\title{
Supervisory control based on minimal cuts and Petri net sub-controllers coordination
}

\author{
Sadok Rezig, Zied Achour, Nidhal Rezg \& Mohamed-Ali Kammoun
}

To cite this article: Sadok Rezig, Zied Achour, Nidhal Rezg \& Mohamed-Ali Kammoun (2016) Supervisory control based on minimal cuts and Petri net sub-controllers coordination, International Journal of Systems Science, 47:14, 3425-3435, DOI: 10.1080/00207721.2015.1076903

To link to this article: https://doi.org/10.1080/00207721.2015.1076903

Published online: 20 Aug 2015.

Submit your article to this journal $[\pi$

ЦIl Article views: 85

View Crossmark data $匚$

㓛

Citing articles: 2 View citing articles $\square$ 


\title{
Supervisory control based on minimal cuts and Petri net sub-controllers coordination
}

\author{
Sadok Reziga, ${ }^{a}$, Zied Achour ${ }^{a, b}$, Nidhal Rezg ${ }^{a, b, b}$ and Mohamed-Ali Kammoun ${ }^{a}$

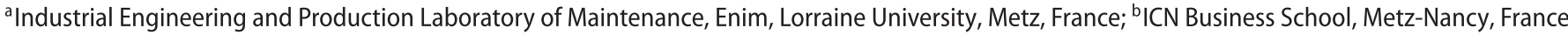

\begin{abstract}
This paper addresses the synthesis of Petri net (PN) controller for the forbidden state transition problem with a new utilisation of the theory of regions. Moreover, as any method of control synthesis based on a reachability graph, the theory of regions suffers from the combinatorial explosion problem. The proposed work minimises the number of equations in the linear system of theory of regions and therefore one can reduce the computation time. In this paper, two different approaches are proposed to select minimal cuts in the reachability graph in order to synthesise a PN controller. Thanks to a switch from one cut to another, one can activate and deactivate the corresponding PNcontroller. An application is implemented in a flexible manufacturing system to illustrate the present method. Finally, comparison with previous works with experimental results in obtaining a maximally permissive controller is presented.
\end{abstract}

ARTICLE HISTORY

Received 25 December 2014 Accepted 23 July 2015

\section{KEYWORDS}

Petri nets; theory of regions; discrete event systems; supervisory control; minimal cuts

\section{Introduction}

The previous research works in supervisory control led to the application of state feedback control to prevent the system to avoid some states in order to assure a desired behaviour. The first investigations developed for discrete event systems(DES)led Ramadge and Wonham (Ramadge \& Wonham, 1987; Ramadge \& Wonham, 1989) to the great results on the existence and synthesis of supervisors for DES. It was shown that if a set $M$ of controllable legal markings, i.e. $\forall \boldsymbol{M} \in M$ there is no reachable forbidden marking from a sequence containing uncontrollable transitions, and if this set is expressed by $\boldsymbol{n}_{\boldsymbol{c}}$ linear inequalities constraints [(or generalised mutual exclusion constraints(GMEC)] then the Petri net (PN)has a solution and the behaviour is maximum permissive (Giua, DiCesare, \& Silva, 1992).

The proposed method of Ghaffari, Rezg, and Xie (2002a) and Ghaffari, Rezg, and Xie (2002b) in a supervisory control problem is based on the theory of regions (Badouel \& Darondeau, 1998). This theory is represented by a linear system(LS) composed of three kinds of conditions, its resolution leads to the synthesis of a PN controller. However, the discussed LS may contain state equations that form a convex combination of other states which make it impossible to resolve. Furthermore, the combinatorial explosion of states in the generated graph complicates the computation of PN supervisors. In this work, the application of the theory of regions is restricted in minimal cuts (MC) selected with specific criteria and not on the graph. Each monitor supervises one $\mathrm{MC}$ in the reachability graph. Therefore, there is a coordination between PN controllers, thanks to a Boolean control function that activates and deactivates the monitors.

Some research has been conducted to synthesise PN supervisors under some bounded assumptions to enforce disjunctive constraints called generalised linear constraints (Iordache \& Antsaklis, 2007). Moreover, situations may exist where the number of places of the least-restrictive controllers was exponentially related to the size of the specification. Iordache, $\mathrm{Wu}$, Zhu and Antsaklis (2013) introduced an enhanced approach that generates PN monitors, in which the number of places is linearly related to the size of the specification. However, in these works, the PN controller is designed under fully controllability. In addition, Haoxun (2000) described a method for constructing controller by forcing the net to obey the linear inequality constraints. Thus, the computation of its PN controller is based on the concept of minimal support S-decreases, but the monitor place is an extended PN. Furthermore, Ru, Cabasino, Giua, and Hadjicostis (2012) synthesised a PN supervisor under arbitrary forbidden state specifications. Indeed, the control policy is determined by checking certain controllable transitions. If it will lead to a subsequent reduced marking that belongs to the forbidden marking, the supervisor disables the corresponding controllable transition.

Our new control policy is focused on the research carried on the utilisation of the theory of regions that has been recognised as the powerful method of deadlock prevention policy for obtaining maximally permissive controllers. Many research works sought to lower the computational cost of the theory of regions; Li, Zhou, and Jeng (2008) utilised an approach called siphons control before using the theory of regions to calculate the PNcontrollers. Furthermore, Huang and Pan (2011) proposed another issue for deadlock prevention to decrease the number of reachability condition equations of the theory of regions in order to facilitate the computation of the supervisors. Their experimental results seem to be the most efficient method in the deadlock prevention policy compared with the works of Uzam and Zhou (2007), Uzam (2009), Park and Reveliotis (2000) and Qin et al. (2012).

In order to reduce the resolution complexity of the theory of regions, this work propose a new method based on MCs (Rezig, 
Achour, \& Rezg, 2014; Rezig, Achour, Rezg, \& Kammoun, 2014), which allows on the one hand to minimsze the total number of equations inLSand the time calculation, and on the other hand to avoid the combinatory explosion of states.

The rest of the paper is structured as follows: Section 2 depicts the background of the theory of regions and PN tools. Section 3 introduces the concept of MCs in the reachability graph for synthesising supervisors. The switch between PNcontrollers is proposed in Section 4 due to the activation and deactivation of supervisors. A flexible manufacturing system(FMS)is presented in Section 5 in order to illustrate the contribution of the new method. Finally, Section 6 presents the comparison of our new method, which will be carried out with the classical approach of the theory of regions (Ghaffari, Njima, Rezg, \& Xie, 2002) to the recent method of Huang and Pan (2011).

\section{Preliminaries}

A PN is a bipartite graph $N=(P, T$, Pre, Post) where $P$ is a set of places, $T$ is a set of transitions with $T=T_{c} \cup T_{u}$ the union of controllable and uncontrollable transitions (Murata, 1989). Pre $: P \times T \rightarrow \mathbb{N}$ is the pre-incidence function that specify weighted arcs from places to transitions, Post $: P \times T \rightarrow \mathbb{N}$ is the post-incidence function that specify weighted arcs from transitions to places, where $\mathbb{N}$ is the set of nonnegative integers. Let $p^{(t)}\left(\right.$ respectively $\left.{ }^{(t)} p\right)$ be the set of output transitions (respectively input transitions) of the place $p$ and $t^{(p)}$ (respectively $\left.{ }^{(p)} t\right)$ the set of output places (respectively input places) of transition $t$. Let $G\left(N, M_{0}\right)$ be the reachability graph constructed from its initial marking $M_{0} . M$ denote by the set of markings $M \in$ $G\left(N, M_{0}\right)$. A transition $t \in T$ is enabled from a marking $M$ (denoted by $M[t>$ ) if and only if $M \geq \operatorname{Pre}(., t)$. An enabled transition $t$ may fire yield a new marking $M^{\prime}$, such that $M=$ $M+C(., t)$, this can be denoted by $M\left[t>M^{\prime}\right.$. A marking $M^{\prime}$ is reachable from a marking $M$ if there exists a firing sequence $\sigma=t_{1}, t_{2} \ldots t_{n}$ transforming $M$ into $M^{\prime}$. By firing a sequence $\sigma$, any marking $M$ reachable from $M_{0}$ satisfies the following state equation of $P N: M=M_{0}+C \vec{\sigma}$, where $\vec{\sigma}: T \rightarrow \mathbb{N}$ a vector of nonnegative integers is called the occurrence of transition $t_{i}$ in $\sigma$ and $C$ is the incidence matrix of a marked $P N$. $C\left(p_{i}, t_{j}\right)=w\left(t_{j}, p_{i}\right) \mathrm{Ift}_{j} \in{ }^{(t)} P_{i}, C\left(p_{i}, t_{j}\right)=-w\left(p_{i}, t_{j}\right)$ ift $t_{j} \in$ $p_{i}{ }^{(t)}$, else 0 , where $w: F \rightarrow \mathbb{N}$ is a valuation function of arcs. $(F \subseteq(T * P) \cup(P * T)$ : finite set of $\operatorname{arcs})$. A PN is $k$-bounded if the number of tokens in each place does not exceed $k$. A PN is live if every transition is live. A PN is reversible if from any reachable state $M$ there is an enabled sequence to return to $M_{0}$. Considering a set of legal markings, such admissible states correspond to a set of constraints GMEC $(\vec{w}, K)$ which defines the set of states (Giua et al., 1992): $M_{(\vec{w}, k)}=\left\{M \in \mathrm{M} \mid \vec{w}^{T} \leq k\right\}$.

In certain cases, the control specification cannot be expressed by GMEC. Therefore, we developed in our previous work (Rezg, Rezig, Kammoun, \& Achour, 2014) a new type of control specification constraint $(\vec{v}, C)$ which defines a set of legal sequences of transitions of transitions called admissible paths constraints:

$$
T_{(\vec{v}, C)}=\left\{\sigma \in \sigma^{*} \mid \vec{v}^{T} \leq C\right\} .
$$

The application of the theory of regions allows the synthesis of the PNcontroller to add to the initial PN.The control place $P_{c}$ with initial marking $M_{0}\left(P_{c}\right)$ and its incidence vectors $C\left(P_{c},.\right)$ has to satisfy the reachability conditions, i.e

$$
M_{0}\left(P_{c}\right)+C\left(P_{c}, .\right) \vec{\Gamma}_{M} \geq 0
$$

Furthermore, each $P_{c}$ to add should solve at least one forbidden event $(M, t)$ in the set $\Omega$ of prohibited state transitions:

$$
M_{0}\left(P_{c}\right)+C\left(P_{c}, .\right) \vec{\Gamma}_{M}+C\left(P_{c}, t\right)<0
$$

Besides, $P_{c}$ has to fulfil cycle equations:

$$
\sum_{t \in T} C\left(P_{c}, t\right) \cdot \vec{\sigma}[t]=0, \forall \sigma \in R
$$

\section{$\vec{\sigma}[t]$ is the algebraic sum of occurrences of $t$ in $\sigma$.}

The(LS)composed of Equations (3)-(5) is used to determine $P_{c}$. Notably, many event separation instances may obtain the same solutions. As a result, the number of $P_{c i}$ will be much smaller than the set of a prohibited state transitions $\Omega$.

Aside from the combinatorial explosion problem in the theory of regions, the convex combination of states equations can be found in LS which make its resolution impossible as in the following example (i.e. see Figure 1). By writing the following LS, the theory of regions is insoluble and the synthesis of the PN

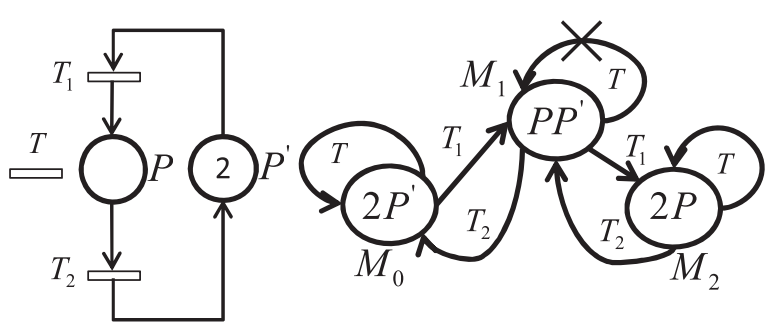

$a$

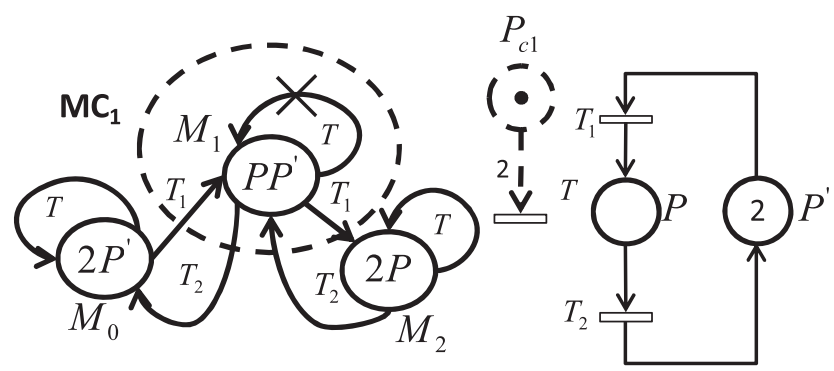

b

Figure 1. An insoluble problem with the classical approach of the theory of regions. a. Insoluble problem with the classical approach. b. Resolution of the insoluble problem. 


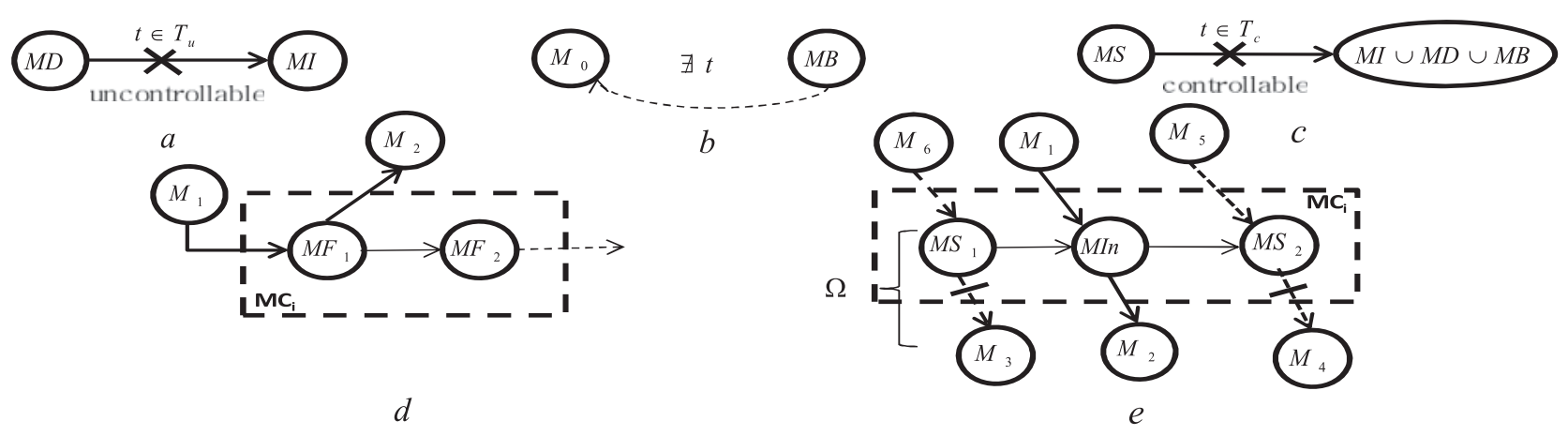

Figure 2. Six kinds of markings. a. Dangerous and forbidden state. b. Blocking state. c. Source state. d. Frontier state. e. Intermediate state.

controller is impossible:

LS : $\left\{\begin{array}{l}M_{0}\left(P_{c}\right)=M_{0}\left(P_{c}\right) \geq 0 \\ M_{1}\left(P_{c}\right)=M_{0}\left(P_{c}\right)+C\left(P_{c}, T_{1}\right) \geq 0 \\ M_{2}\left(P_{c}\right)=M_{0}\left(P_{c}\right)+2 C\left(P_{c}, T_{1}\right) \geq 0 \\ M_{0}\left(P_{c}\right)+C\left(P_{c}, T_{1}\right)+C\left(P_{c}, T\right)<0 \\ C\left(P_{c}, T\right)=0 \\ C\left(P_{c}, T_{1}\right)+C\left(P_{c}, T_{2}\right)=0\end{array}\right.$.

Indeed, it is clear that $M_{1}\left(P_{c}\right)$ is a convex combination of $M_{0}\left(P_{c}\right)$ and $M_{2}\left(P_{c}\right)$; (i.e. $M_{1}\left(P_{c}\right)=\frac{1}{2} M_{0}\left(P_{c}\right)+\frac{1}{2} M_{2}\left(P_{c}\right)$ ). Therefore, the event $\left(M_{1}, T\right)$ cannot be forbidden with the application of theory of regions.

In the following section, an efficient method to reduce the total number of equations inLS will be introduced. For convenience, our proposed new method will follow the above interpretation of the theory of regions.

\section{Minimal cuts in a reachability graph}

\subsection{Problem setting}

This work is focused on the reduction of the computational cost of the theory of regions by minimising the number of equations in its LS; hence, one can automatically save computing time of the theory of regions. Therefore, a new concept of MC is introduced in the reachability graph to achieve these aims by two approaches.

Definition 1: A state is said forbidden state if it does not respect a given specification $(\vec{w}, K)$. (i.e. see Figure $2(\mathrm{a})$ ). Let $M I$ be the set of forbidden states: $M I=\left\{M \in M \mid M \neq M_{(w, k)}\right\}$.

Definition 2: The set of event separation instances that the supervisor has to disable is

$$
\Omega=\left\{(M, t) \mid \exists M\left[t>M ;^{\prime} \wedge M \in G\left(N, M_{0}\right) \wedge M^{\prime} \in M I\right\}\right.
$$

(i.e. see Figure $3(c)$ ).

Definition 3: A state is said a dangerous state if it leads to the forbidden state by firing uncontrollable transitions. Let $M D$ be the set of dangerous states: $M D=$ $\left\{M \in G\left(N, M_{0}\right) \mid \exists t \in T_{u}, \exists \sigma \in T_{u}^{*}, M\left[\sigma>M^{\prime} \wedge M^{\prime}\right.\right.$ $\left[t>M^{\prime \prime} \wedge\left(M^{\prime}, t\right) \in \Omega\right\}$ (Figure 2(a)).

Definition 4: A state is said a blocking state if it does not belong to a live sub-graph (LSG); LSGis a sub-graph of the reachability graph $G\left(N, M_{0}\right)$ containing all the states $M \in G\left(N, M_{0}\right)$ leading to $M_{0}$. Let $M B$ be the set of blocking states:

$$
M B=\left\{M \in G\left(N, M_{0}\right) \mid \nexists \sigma \epsilon T^{*}, M\left[\sigma>M_{0}\right\}\right. \text { (Figure 2(b). }
$$
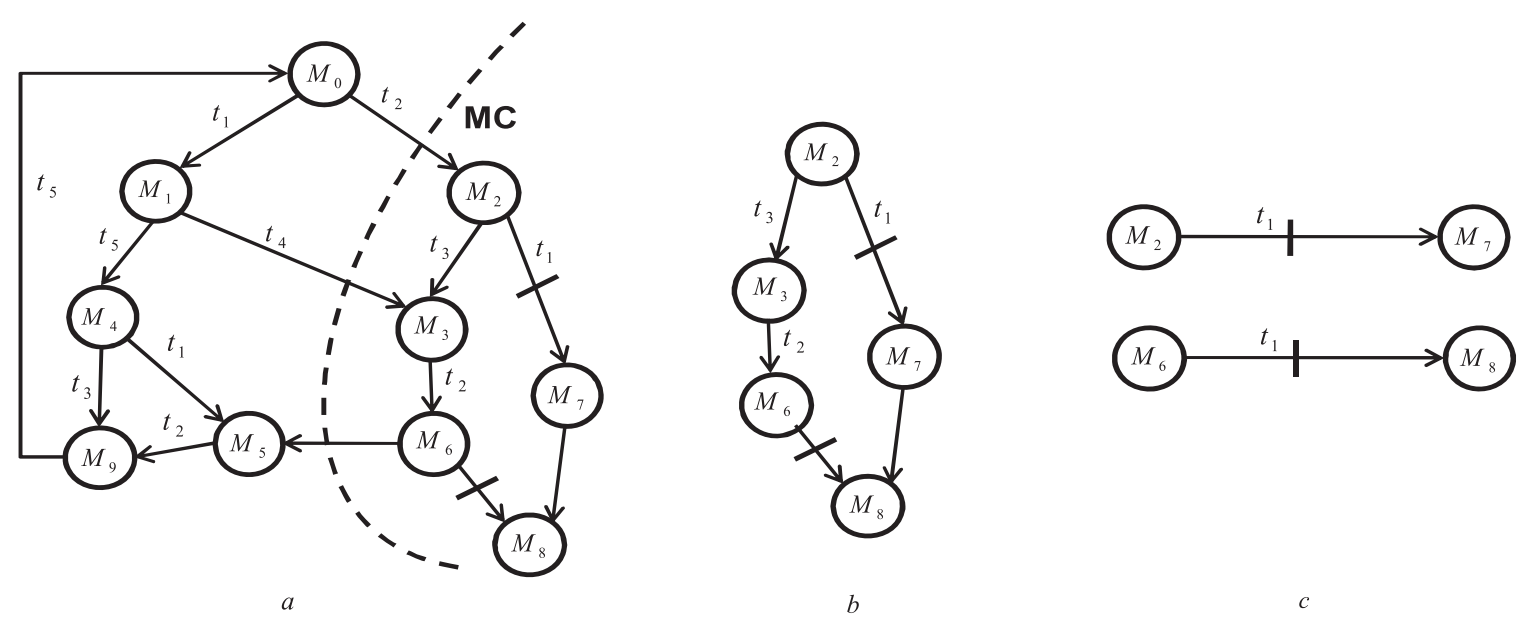

Figure 3. Example of minimal cut, MC. a. Reachability graph. b. MC. c. Correlated event separation instances with $t_{1}$. 
Definition 5: A state is said a source state if it is an admissible state in $M C$ and from which we prohibit a forbidden state transition $(M, t)$ of $\Omega$. Let $M S_{i}$ be the set of source states (Figure 2(c)): $\quad M S_{i}=\left\{M \in G\left(N, M_{0}\right) \mid \exists\left(M\left[t>M^{\prime}\right), M \in \Omega, \quad M^{\prime} \in\right.\right.$ $\left.(M I \cup M D \cup M B), t \in T_{c}\right\}$.

Definition 6: A state is said frontier if it is a state from which we activate (deactivate) a MC. Let $M F_{i}$ be the set of frontier states (Figure 2(d):

$$
M F_{i}=\left\{M \in M C_{i} \mid \exists M_{i}[t>M), M_{i} \notin M_{i}\right\} .
$$

\subsection{Approach 1: minimal cut with correlated event separation instances}

The identification of a minimal cut $M C_{i}$ in the reachability graph $G\left(N, M_{0}\right)$ is characterised by the correlation of event separation instances. Nevertheless, the application of the theory of regions in control synthesis will be restricted in $M C_{i}$, and will generate one control place $P_{c i}$ for each $\mathrm{MC}_{i}$.

Definition 7: A minimal cut $\mathrm{MC}_{i}$ is a zone of the reachability graph $G\left(N, M_{0}\right)$ with minimal number of nodes which is identified by one control place $P_{c i}$, ensures the reachability of all markings contained in $\mathrm{MC}_{i}$ and solves one or more event separation instances (i.e. see Figure 3(a) and 3(b)): $M C_{i}=M S_{i} \cup$ $\left\{M\left[\sigma>M^{\prime} \mid M, M^{\prime} \in M S_{i}, \sigma \in T^{*}\right\}\right.$

Definition 8: Two event separation instances are correlated if their corresponding transition to prohibit is the same. Let $\Omega_{11}=$ $\left(M, t_{i}\right), \quad \Omega_{12}=\left(M^{\prime}, t_{j}\right) \in \Omega$, if $t_{i}=t_{j}$ then $\Omega_{11}$ and $\Omega_{22}$ are correlated (i.e. see Figure 3(c)).

Definition 9: A state is said an intermediate state if it is a frontier state belonging $M C_{i}$ and it is not a source state: $M I n_{i}=$ $\left\{M \in M C_{i} \mid M \epsilon M F_{i} / M S_{i}\right\}$ (Figure 2(e)).

Proposition 1: An initial source state $M_{0 i}$ of a minimal cut $\mathrm{MC}_{i}$ belongs to the set of source states $M S_{i}$ and is generated by the smallest transition vector $x$ in the reachability graph $G\left(N, M_{0}\right)$ from its initial state $M_{0}$.

Definition 10 (Darondeau \& Xie, 2003): A transition vector $x$ of a state $M \in G\left(N, M_{0}\right)$ is said the smallest transition vector if its corresponding state $M$ is calculated with the smallest transition firing sequence $\sigma$ from $M_{0}$, (i.e. the smallest $i$, with $=|\sigma|$ : number of transitions in $\sigma$ called its length).

Proof: Let $x$ and $x^{\prime}$ be the respective transition vectors of $M$ and $M^{\prime}\left(M, M^{\prime} \in G\left(N, M_{0}\right)\right)$ and let $C$ be the incidence matrix of a $P N . \vec{\sigma}$ and $\vec{\sigma}^{\prime}$ are the associated transition firing vectors of $M$ and $M^{\prime}$, such that: $M=M_{0}+C . \vec{\sigma}$ and $M^{\prime}=M_{0}+C . \vec{\sigma}^{\prime}$. From Darondeau and Xie (2003), if $\sigma^{\prime} \geq \sigma$ it exists a transition firing sequence: $\sigma^{\prime \prime}=\sigma^{\prime}-\sigma \mid M\left[\sigma^{\prime \prime}>M^{\prime}\right.$ then $x$ is smaller than $x^{\prime}$.

The identification of MCs is described in the following algorithm 1 .

Algorithm 1 : Identification of $M C$ in $G\left(N, M_{0}\right)$.

Let $Q$ be a given control specification and $M A=$ $(M I \cup M D \cup M B)$ the set of markings to avoid.

(1) Determine sets of states and state transitions, respectively, $M I$ and $T F$ that does not respect $Q$.
(2) Specify the event separation instances:

$T_{f}=\left\{\left(M\left[t>M^{\prime}\right), M \in G\left(N, M_{0}\right), M_{\prime} \in M I\right\} ; T F=T F \cup\right.$ $T_{f}$.

(3) Identify the set of dangerous states $M \operatorname{Din} G\left(N, M_{0}\right)$.

(4) Identify controllable transitions leading to $M B$.

(5) Determine the set of blocking states.

(6) Identify controllable transitions leading to $M B$

(7) Specify the event separation instances:

$\Omega=\left\{\left(M t>M^{\prime}\right), M \in G\left(N, M_{0}\right), t \in T_{c}, M^{\prime} \in M A\right\}$.

(8) Define the set of source states:

$M S=\left\{\left(M \in G\left(N, M_{0}\right) \mid \exists(M, t) \in \Omega, t \in T_{c}\right\}\right.$.

(9) If $M \notin(M S \cap M B)$ then go to the next step. Else eliminate $M[t>$ of $\Omega$ and go to step 5 .

(10) Determine each set $\Omega_{i}$ of correlated event separation instances.

(11) Regroup source states $M S_{i}$ from each set $\Omega_{i}$ and paths connecting them in $\mathrm{MC}_{i}$.

(12) Define initial source state $M S_{i}$ from $\mathrm{MC}_{i}$.

END

In the first 9 steps, the avoided states and event separation instances are identified. Thus, one can determinate the set of event separation instances $\Omega=$ $\left\{\left(M\left[t>M^{\prime}\right), M \in G\left(N, M_{0}\right), t \in T_{c}, M^{\prime} \in M A\right\}\right.$. In the following steps, MC are grouped according to the correlation of event separation instances in $\mathrm{MC}_{i}$.

Remark 1: The application of the theory of regions in the method of Ghaffari et al. (2002a) in control synthesis considers the entire $G\left(N, M_{0}\right)$, however in this new method, the union of $\mathrm{MC}$ does not form the entire reachability graph: $\cup \mathrm{MC}_{i} \subset$ $G\left(N, M_{0}\right)$, which approves the reduction of computational cost of the theory of regions.

Theorem 1: If a marking enters a $\mathrm{MC}_{i}$ by any source state $M \in$ $M S_{i} \mid M \neq M_{0 i}$, the initial marking of $P_{c i}$ does not need to be recalculated and the theory of regions remains valid.

Proof: (reduction ad absurdum): Let $M C_{i}$ be a minimal cut in a reachability graph $G\left(N, M_{0}\right)$, correlated by transition $t_{1}$ (Figure 4) and $M I_{i}$ the set of forbidden states, $M I_{i}=$ $\left\{M^{\prime}, M^{\prime \prime}\right\}$. Hypothesis: If a marking enters a $\mathrm{MC}_{i}$ by $M \in$ $M S_{i} \mid M \neq M_{0 i}$, such that $\left(M_{0 i} \rightarrow t_{1} M^{\prime}\right) ;\left(M \rightarrow t_{1} M^{\prime \prime}\right)$, then with the theory of regions already calculated from $M_{0 i}$, transition $t_{1}$ cannot be prohibited if a marking enters a $\mathrm{MC}_{i}$ by $M$ and one can write: $M^{\prime \prime}\left(P_{c i}\right)=M\left(P_{c i}\right)+C\left(P_{c i}, t_{1}\right) \geq$

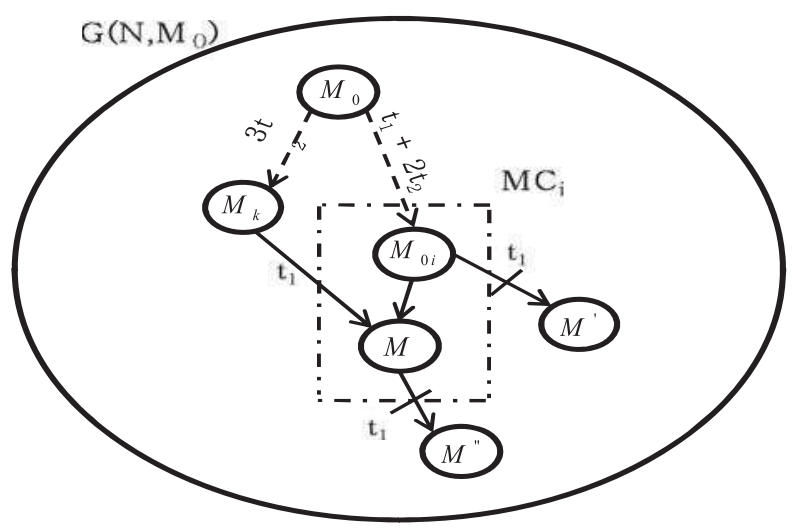

Figure 4. Example of minimal cut. 
0 and the theory of regions is not valid.Suppose that a marking $M_{k}$ enters a $\mathrm{MC}_{i}$ starting byt $t_{1}$, such that $M_{k}\left(P_{c i}\right)=$ $M_{0}\left(P_{c i}\right)+3 C\left(P_{c i}, t_{2}\right), M=M_{k}+C\left(P_{c i}, t_{1}\right)=M_{0}\left(P_{c i}\right)+$ $3 C\left(P_{c i}, t_{2}\right)+C\left(P_{c i}, t_{1}\right)$.Since $M^{\prime \prime}\left(P_{c i}\right)=M\left(P_{c i}\right)+C\left(P_{c i}, t_{1}\right)=$ $>M^{\prime \prime}\left(P_{c i}\right)=M_{0}\left(P_{c i}\right)+2 C\left(P_{c i}, t_{1}\right)+3 C\left(P_{c i}, t_{2}\right) \geq 0$ or $M_{0 i}$ is the state calculated with the smallest transition vector, so $M^{\prime \prime}$ can be expressed by $M_{0 i}$ of $M C_{i}$. From Figure 4, $M_{0 i}\left(P_{c i}\right)=M_{0}\left(P_{c i}\right)+C\left(P_{c i}, t_{1}\right)+2 C\left(P_{c i}, t_{2}\right) ; M^{\prime \prime}\left(P_{c i}\right)=$ $M_{0 i}\left(P_{c i}\right)+C\left(P_{c i}, t_{1}\right)+C\left(P_{c i}, t_{2}\right) \geq 0$. This is absurd; indeed, the application of the theory of regions from initial state $M_{0 i}$ of $\mathrm{MC}_{i}$ gives $M^{\prime \prime}\left(P_{c i}\right)=M_{0 i}\left(P_{c i}\right)+C\left(P_{c i}, t_{1}\right)+C\left(P_{c i}, t_{2}\right)<0$, whereas if a marking enters a $\mathrm{MC}_{i}$ by any state $M \in M S_{i}$, initial marking of control place $M_{0 i}\left(P_{c i}\right)$ does not need to be recalculated and the theory of regions remains valid.

Once $M C_{i}$ are determined, relations 3, 4 and 5 make it possible to find $P_{c i}$, the following algorithm summarises the calculation of the $\mathrm{PN}$ supervisor.

Algorithm 2 : Synthesis of $P_{c i}$ for each $\mathrm{MC}_{i}$

Let $M_{0 i}$ be the initial state of $\mathrm{MC}_{i}$, and $n_{i}=\operatorname{card}\left(\Omega_{i}\right)$.

(1) Initialization, $i:=1$.

(2) If $n_{i}=1$.

(2.1) Write the reachability equation of $M_{0 i}$.

(2.2) Write the event separation instance equation of $\left(M_{0 i}[t>\right.$ $\left.M^{\prime}\right) \in \Omega_{i}$.

(2.3) Solve the linear system $L S_{i}$ and $\operatorname{let}\left(M_{0 i}\left(P_{c i}\right), C\left(P_{c i},.\right)\right) b e$ the solution of $\mathrm{MC}_{i}$.

(3) Else if $n_{i}>1$ :

(3.1) Identify independent equations of basic cycles using the Gauss method.

(3.2) Write the reachability equations of $M \in \mathrm{MC}_{i}$ from $M_{0 i}$.

(3.3) Write the event separation equations of the set $\Omega_{i}$ from $M_{0 i}$.

(3.4) Solve the LS composed of equations of steps 3.1, 3.2 and 3.3. Let $\left(M_{0 i}\left(P_{c i}\right), C\left(P_{c i},.\right)\right)$ be the solution of $M C_{i}$

(4) Eliminate redundant control places.

(5) While $i<n_{i}, i:=i+1$. Return to step 2.

END.

\subsection{Approach 2: minimal cut for any event separation instances}

In this approach, the design of $\mathrm{MC}_{i}$ is only interesting in forbidden state transitions, that does not respect a control specification $Q$.
Definition 11: $\quad \mathrm{MC}_{i}$ is a zone of $G\left(N, M_{0}\right)$ with minimal number of nodes which identify a PN supervisor, and is only interesting in event separation instances. $M C_{i}=$ $\left\{\left(M\left[t>M^{\prime}\right) \mid M \in M S, M^{\prime} \in M A\right\}\right.$. (i.e. see Figure 5).

Next, the design of MCs with any event separation instances is described in Algorithm 3. Indeed, steps 1-9 which identify the forbidden state and the forbidden state transition are the same as in Algorithm 1; the difference is the way to construct $\mathrm{MC}_{i}$ defined in steps 10-12.

Algorithm 3 : Identification of $\mathrm{MC}_{i}$ in $G\left(N, M_{0}\right)$

Let $T_{a}(M)$ be a set of $\operatorname{arcs}$ of a source state $M \in M S$

(10) Determine for each $M \in M S$, arcs:

$T_{a}(M)=\left\{t \in T \mid\left(M\left[t>M^{\prime \prime}\right), M \in M S, M^{\prime \prime} \notin M A\right\}\right.$

(11) Associate each $M \in M S a n d$ its event separation Instances $\left(M\left[t>M^{\prime} M \in M S \mid M^{\prime} \in M A\right)\right.$ a $M_{i}$.

(12) If ( $M^{\prime} \in M A \mid M\left[t>M^{\prime}, M \in M S\right)$ is the

common state between minimal cut $M C_{i}$,

and $t \in T \mid\left(M\left[t>M^{\prime}\right) \notin T_{a}(M)\right.$ then merge

Minimal cuts $M C_{i}$.

END.

The most attractive advantage of the proposed approach is the design for each source states with its event separation instances a $\mathrm{MC}_{i}$ without basic cycles. However, the fusion of $\mathrm{MC}$ will reduce the number of $\mathrm{MC}_{i}$, therefore the number of $\mathrm{PN}$ controllers is reduced.

The control synthesis is described in the following Algorithm 4.

Algorithm 4 : Synthesis of PN controller for $\mathrm{MC}_{i}$

Let $\mathrm{MC}$ be the set of minimal cuts, $M_{0 i}$ the initial state of each $\mathrm{MC}_{i}$ and $n_{i}=\operatorname{card}\left(\Omega_{i}\right)$.

(1) Initialization, $i:=1$

(2) If $n_{i}=1:$

(2.1) Write the reachability equation of initial state $M_{0 i}$ of $\mathrm{MC}_{i}$.

(2.2) Write the event separation instance equation of $M_{0 i}[t>$ $M^{\prime}, M^{\prime} \in M A$.

(2.3) Solve $L S_{i}$ and let $\left(M_{0 i}\left(P_{c i}\right), C\left(P_{c i},.\right)\right)$ be the solution of $\mathrm{MC}_{i}$.

(3) Else if $n_{i}>1$ :

(3.1) Write the reachability equation of initial state $M_{0 i}$ of $\mathrm{MC}_{i}$.
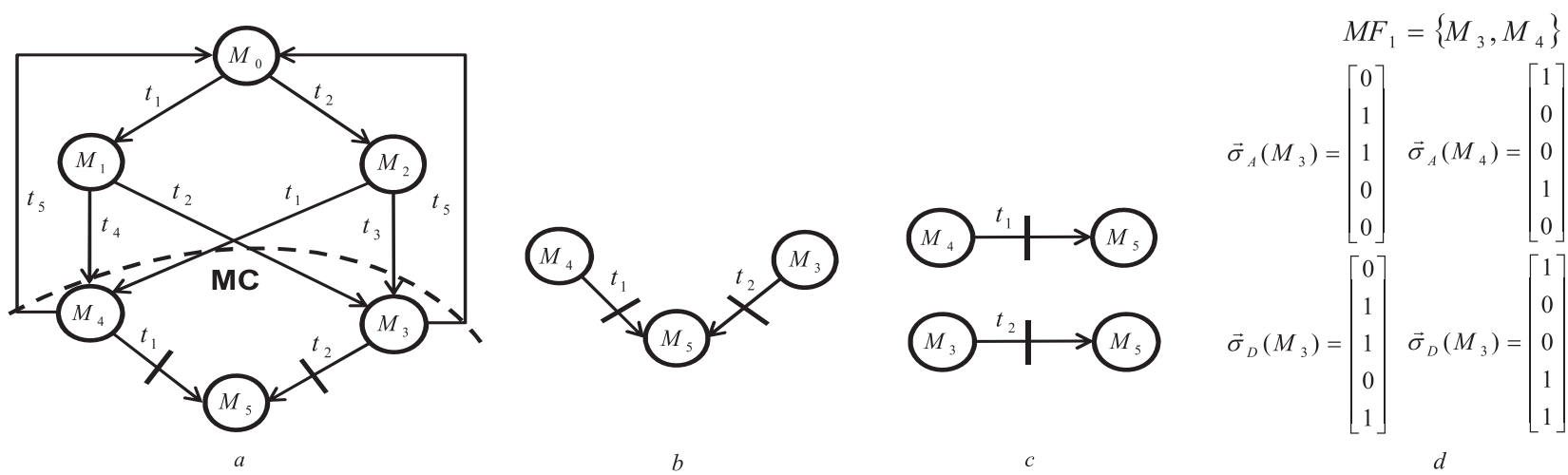

$b$

Figure 5. Example of minimal cut. a. Reachability graph. b. Minimal cut, MC. c. Event separation instances. d. Activation/deactivation vectors. 
(3.2) Write the event separation instance equations from $M_{0 i}$.

(3.3) Solve $L S_{i}$ and let $\left(M_{0 i}\left(P_{c i}\right), C\left(P_{c i},.\right)\right)$ Be the solution of $\mathrm{MC}_{i}$

(4) Eliminate redundant control places.

(5) While $i<n_{i}, i=+1$;

Return to step 2.

END.

Using the same example of Figure 1, one can apply our policy to write the following LS: $M_{01}\left(P_{c 1}\right) \geq 0 ; \quad M_{01}\left(P_{c 1}\right)+$ $C\left(P_{c 1}, T\right)<0$. The resolution of LS gives $M_{01} \quad\left(P_{c 1}\right)=$ $1 ; C\left(P_{c 1}, T\right)=-2$. The controlled $\mathrm{PN}$ is given in Figure $1(\mathrm{~b})$.

Despite the reduction of the computation time and the equations number, our proposed method can solve insoluble problems with the original theory of regions. It is also required to calculate the activation/deactivation vectors of $M F_{1}=\left\{M_{1}\right\}$ to enable/disable $P_{c 1}$.

After synthesising the $\mathrm{PN}$ controllers for each $\mathrm{MC}_{i}$, one can determine the Boolean control function $\boldsymbol{F}_{\boldsymbol{B} \boldsymbol{c}}$ that allows the activation and the deactivation of supervisors. Thus, the role of each monitor $P_{c i}$ is to control its zone $\left(\mathrm{MC}_{i}\right)$ in the reachability graph. The activation/deactivation vectors and the control Boolean function are described in the following Section 4.

\section{The switch and coordination between Petri net controllers}

In this section, an alternation policy of PN controllers is developed through a switch between them in the reachability graph by activating and deactivating the supervisor $P_{c i}$ of each $\mathrm{MC}_{i}$. Additionally, a Boolean control function will be introduced.

\subsection{Activation and deactivation vectors}

After the identification of $\mathrm{MC}_{i}$ and $P_{c i}$ in the reachability graph, we proceed to the step of activation/deactivation of supervisors. Once a marking enters $\mathrm{MC}_{i}$, each $P_{c i}$ has the task to control its zone in the reachability graph. The $\mathrm{PN}$ controller is initially inactive (active) until a marking enters (exits) a $\mathrm{MC}_{i}$ by its frontier states. Here, one can formally define the activation/deactivation vector.

Definition 12: We associate for each $M \in M F_{i}$ an activation (deactivation) vector, which is a path between $M_{0}$ and $M \in$ $M F_{i}$ by eliminating eventual cycles, which can reactivate (redeactivate) $P_{c i}$.

Activation vector: $\vec{\sigma}_{A}(M)=\sigma \in T^{*} \mid \exists M_{0}[\sigma>M, M \epsilon M F$. Deactivation vector: $\vec{\sigma}_{D} \quad(M)=\sigma \in T^{*} \mid \exists M_{0}[\sigma>$ $M \wedge M\left[t>, M \in M F, t \notin M_{i}\right.$.

Theorem 2: Once a marking entersMC $\mathrm{C}_{i}$ by intermediate states $M \in M I n_{i}$, the initial marking of $P_{c i} \quad$ changes as follows: $M_{0 i} \quad\left(P_{c i}\right)=M_{0 i} \quad\left(P_{c i}\right)+$ $C\left(P_{c i},.\right)\left[\vec{\sigma}_{A}(M)-\vec{\sigma}_{A}\left(M_{0 i}\right)\right]$ (Figure 6).

Proof: The firing sequences $\sigma_{A}\left(M_{0 i}\right)$ and $\sigma_{A}(M)$ are enabled from $M_{0}$ and lead to markings $M_{0 i}$ and $M \in M I n_{i}$ of $\mathrm{MC}_{i}$. Considering the proposition $1 \quad\left(\sigma_{A}\left(M_{0 i}\right)<\sigma_{A}(M)\right)$ and from Darondeau and Xie (2003), $\exists \sigma^{\prime \prime} \mid M_{0 i}\left[\sigma^{\prime \prime}>, \sigma^{\prime \prime}=\right.$ $\left.\sigma_{A}(M)-\sigma_{A}\left(M_{0 i}\right)\right]$. The obtained marking after firing the sequence $\sigma^{\prime \prime}$ from $M_{0 i}$ is $M_{0 i}+C \quad\left[\vec{\sigma}_{A}(M)-\vec{\sigma}_{A}\left(M_{0 i}\right)\right]=$ $\left[M_{0}+C \vec{\sigma}_{A}\left(M_{0 i}\right)\right]+C \quad\left[\vec{\sigma}_{A}(M)-\vec{\sigma}_{A}\left(M_{0 i}\right)\right]=M_{0}+$ $C \quad \sigma_{A}(M)=M . \quad M \in M \operatorname{In}_{i}$. One can deduce that $M_{0}\left[\sigma_{A}\left(M_{0 i}\right) \sigma^{\prime \prime}>M, \quad M \in M I n_{i}\right.$ and $\sigma^{\prime \prime}=\sigma_{A}(M)-$ $\sigma_{A}\left(M_{0 i}\right)$, (i.e. see in Figure 6).

\subsection{Boolean control function}

One can realise that all supervisors can control the system either simultaneously or alternately. More precisely, depending on the process state, the necessary PN controller is activated/deactivated. Here, one can formally define the Boolean control function $\boldsymbol{F}_{\boldsymbol{B} c}$ as follows: $\boldsymbol{F}_{\boldsymbol{B} c}=F\left(\boldsymbol{P}_{\boldsymbol{c} 1}, \boldsymbol{P}_{c 2} . ., \boldsymbol{P}_{\boldsymbol{c} m}\right)=$ $\boldsymbol{P}_{c 1}+\boldsymbol{P}_{c 2}+\ldots \boldsymbol{P}_{c m}=\sum_{i=1}^{m} \boldsymbol{P}_{c i}$

This OR logic function characterises the union of supervisors and takes the value 1 if the system is controlled and 0 otherwise. A simple PN example that models two machines sharing one resource is employed in Figure 7(a). The following GMEC can be satisfied in the reachability graph (Figure $7(\mathrm{~b})$ ) to illustrate the present approach: $M\left(P_{2}\right)+M\left(P_{3}\right)+M\left(P_{5}\right)+M\left(P_{6}\right) \leq 1$. In this example, the supervisors will intervene alternatively: the Boolean control function is $F_{B c}=P_{c 1} P_{c 2}$.

\section{Example}

A physical system composed of two stations in FMS in Ecole Nationale d'Ingenieurs de Metz (ENIM) whose assembly engraved glass with stands, and its PN model are presented in Figure 8(a) and Figure 8(b). The following scenario is applied: the first station modelled by $P_{2}$ represents our selfproduction, while the second station modelled by $P_{3}$ specifies a subcontractor $C_{M}$ and $C_{S}$, respectively, indicate the capacity of three pieces of the machine and the subcontractor. Place $P_{1}$ represents our stock production, $\left(t_{1}, t_{2}\right)$ and $\left(t_{4}, t_{5}\right)$ model, respectively, the events of entry and exit of the pallets in stations through their annexes conveyors, otherwise the pallet can continue its way into the main conveyor by $t_{3}$. Note that only $\left(t_{1}, t_{2}\right)$ are controllable. The constraint to enforce is to keep the marking of the place $P_{2}$ plus the doubled marking of $P_{3}$ less than three tokens: $\boldsymbol{M}\left(\boldsymbol{P}_{2}\right)+2 \boldsymbol{M}\left(\boldsymbol{P}_{3}\right) \leq 3$. This GMEC expresses the following control specification $(Q)$ : the production is entrusted to a subcontractor within the context of sub-trade work. $G\left(N, M_{0}\right)$ contains 13 states (Figure 9).

\subsection{Application of the first approach}

This work proposes an efficient approach to determine MC with minimal number of nodes in which the control synthesis is applied. According to Definition 8, the set $\Omega$ contains five event separation instances correlated by $t_{2}$ or by $t_{1}$ :

$$
\Omega_{1}:\left\{\begin{array}{c}
\left(M_{2}\left[t_{2}>M_{4}\right) ;\left(M_{5}\left[t_{2}>M_{8}\right) ;\right.\right. \\
\left(M_{10}\left[t_{2}>M_{13}\right)\right.
\end{array}\right\}
$$

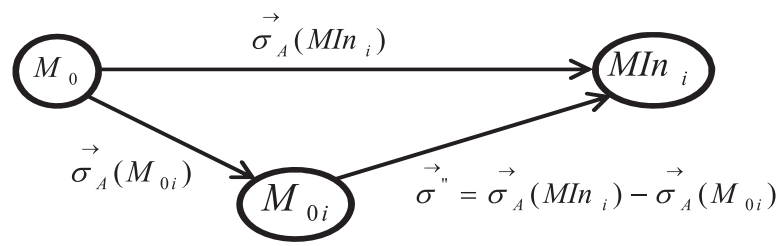

Figure 6. Relation between $M_{0 i}$ and $M / n_{i}$. 


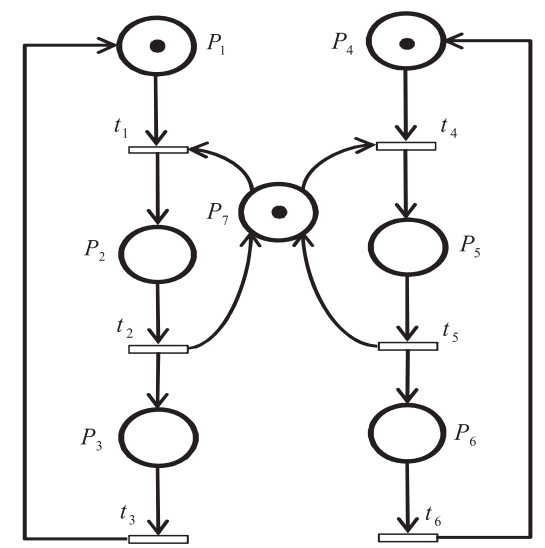

$a$

Figure 7. A simple PN model. a. PN model. b. Reachability graph.

$$
\Omega_{2}:\left\{M_{5}\left[t_{1}>M_{9}\right\}\right.
$$$$
\Rightarrow \mathrm{MC}_{1}:\left\{\begin{array}{c}
\left(M_{2}\left[t_{2}>\right) ;\left(M_{2}\left[t_{1}>\right) ;\left(M_{3}\left[t_{1}>\right) ;\left(M_{5}\left[t_{2}>\right) ;\right.\right.\right.\right. \\
\left(M_{5}\left[t_{5}>\right) ;\right. \\
\left(M_{6}\left[t_{2}>\right) ;\left(\left[t_{1}>\right) ;\left(M_{10}\left[t_{2}>\right)\right.\right.\right.
\end{array}\right\}
$$

\section{$\mathrm{MC}_{2}:\left\{\left(M_{5}\left[t_{1}>\right)\right\}\right.$}

The detailed information of markings of MC is listed in Table 1. Owing to the limitation of paper space; the equations of the theory of regions are not listed.

Let $M_{01}=M_{2}$ and $M_{02}=M_{5}$ be the initial states of $\mathrm{MC}_{1}$ and $\mathrm{MC}_{2}$. Additionally, by applying Algorithm 2, two control places $P_{c 1}$ and $P_{c 2}$ are obtained and shown in Figure 10 when equations (3), (4) and (5) for each $\mathrm{MC}_{i}$ are solved. $P_{c 1}$ : $M_{2}\left(P_{c 1}\right)=M_{01}=1 ; C\left(P_{c 1},.\right)=(-1,-2,0,1,2) \cdot P_{c 2}$ : $M_{5}\left(P_{c 2}\right)=M_{02}=1 ; C\left(P_{c 2},.\right)=(-2,0,0,0,0)$.

According to our new policy, the switch between PN controllers $P_{c 1}$ and $P_{c 2}$ is done by frontier states: $M F_{1}=\left\{M_{2}, M_{3}\right\}, M F_{2}=\left\{M_{5}\right\}$. One can infer that it is only in the state $M_{5}$ that the supervisors are involved simultaneously

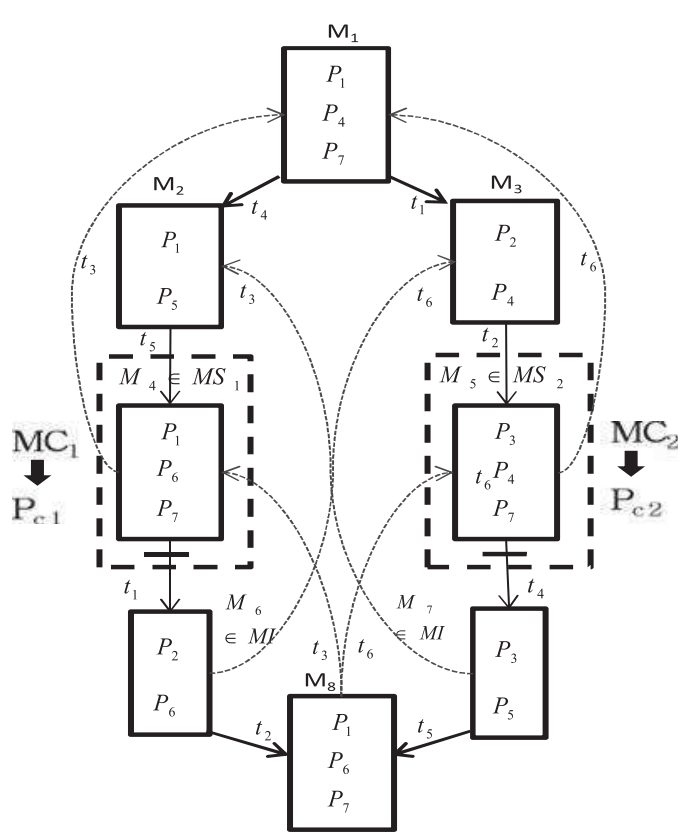

$b$ otherwise they act alternatively. The Boolean control function is $\boldsymbol{F}_{\boldsymbol{B} c}=\boldsymbol{P}_{c 1}+\boldsymbol{P}_{c 2}$.

If marking $M_{1}$ enters $\mathrm{MC}_{1}$ by intermediate state $M_{3}$, the initial marking of $P_{c 1}$ changes as follows: $M_{01} \quad\left(P_{c 1}\right)=$ $M_{01}\left(P_{c 1}\right)+C\left(P_{c 1},.\right) \quad\left[\vec{\sigma}_{A}\left(M_{3}\right)-\vec{\sigma}_{A}\left(M_{2}\right)\right]=2$. To switch between $P_{c 1}$ and $P_{c 2}$, detailed information of activation and deactivation vectors for $P_{c 1}$ and $P_{c 2}$ is listed in Table 2 .

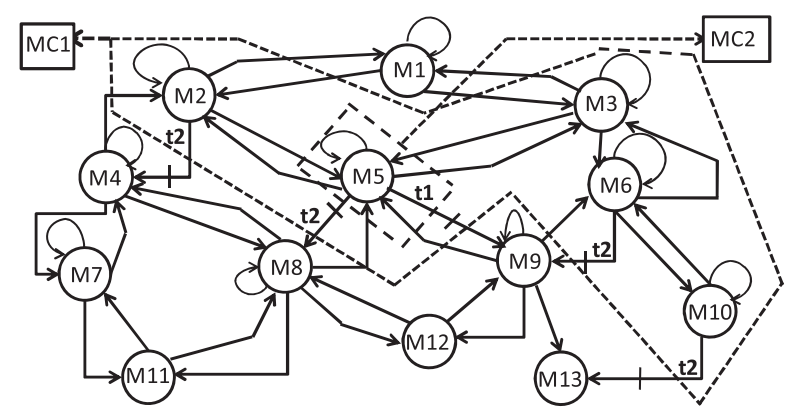

Figure 9. Reachability graph 1 with minimal cuts.
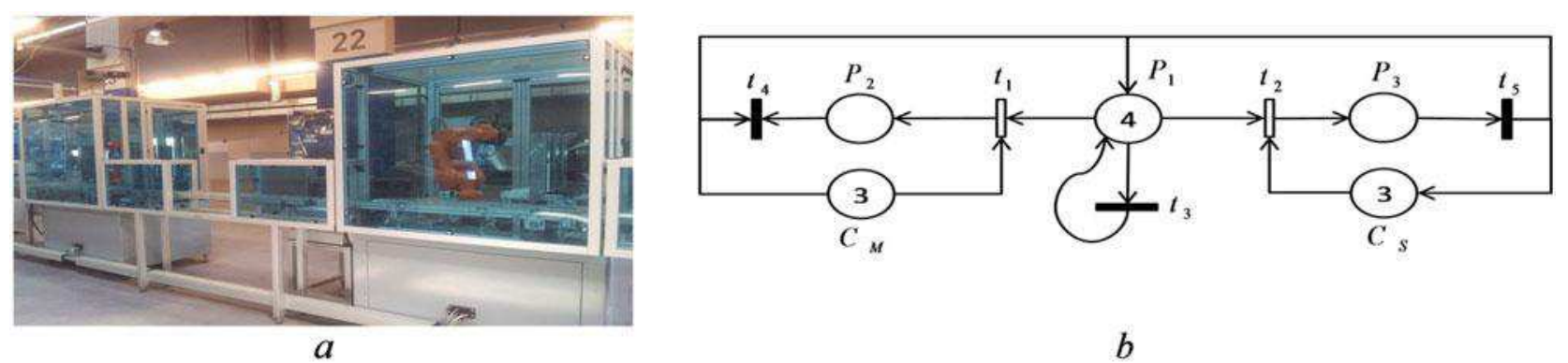

Figure 8. ENIM FMS. a. Experimental environment. b. PN model. 

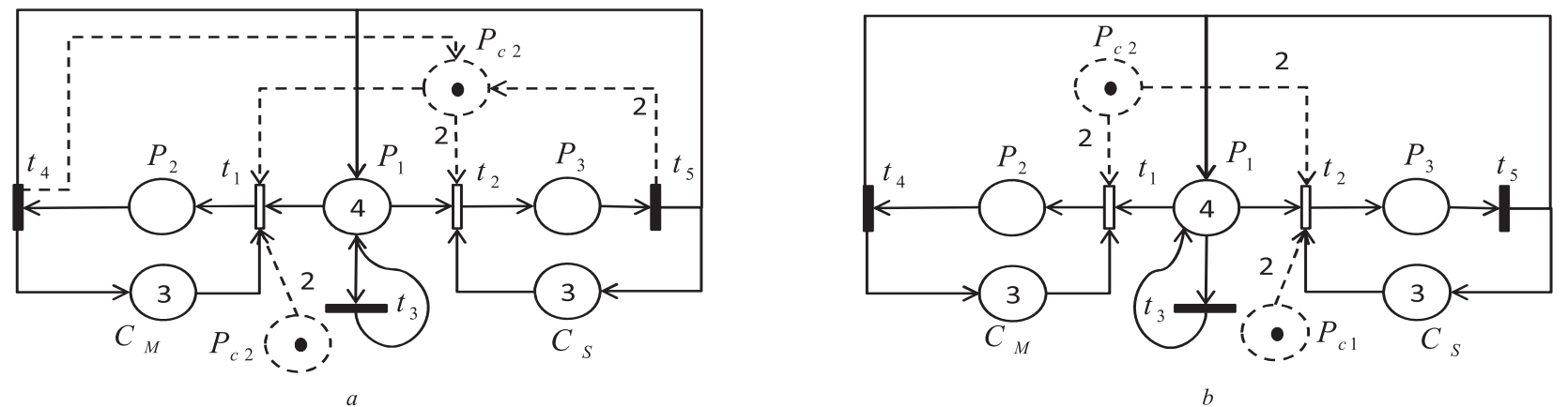

Figure 10. The controlled PN model of FMS. a Controlled PN model 1. b Controlled PN model 2.

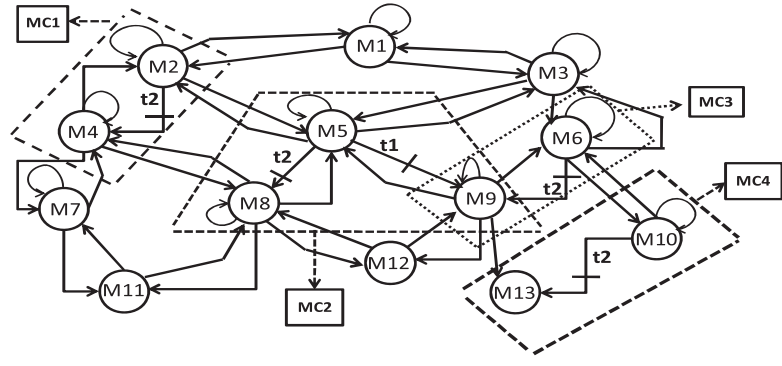

Figure 11. Reachability graph 2 with minimal cuts.

\subsection{Application of the second approach}

The design of MC in this work differs from the last approach and it is only interesting in event separation instances. Next, by using algorithm 3 , one can generate the sources states: $M S$ : $\left\{M_{2} ; M_{5} ; M_{6} ; M_{10}\right\}$ and $\operatorname{arcs} T_{a}\left(M_{i}\right)$ showed in Figure 11.

$$
\begin{aligned}
& T_{a}\left(M_{2}\right):\left\{\left(M_{2}\left[t_{1}>\right) ;\left(M_{2}\left[t_{3}>\right) ;\left(M_{2}\left[t_{5}\right)\right\},\right.\right.\right. \\
& T_{a}\left(M_{5}\right):\left\{\left(M_{5}\left[t_{3}>\right) ;\left(M_{5}\left[t_{4}>\right) ;\left(M_{5}\left[t_{5}\right)\right\}\right\},\right.\right. \\
& T_{a}\left(M_{6}\right):\left\{\left(M_{6}\left[t_{1}>\right) ;\left(M_{6}\left[t_{3}>\right) ;\left(M_{6}\left[t_{4}>\right)\right\},\right.\right.\right. \\
& T_{a}\left(M_{10}\right):\left\{\left(M_{10}\left[t_{3}>\right) ;\left(M_{10}\left[t_{4}>\right)\right\} .\right.\right.
\end{aligned}
$$

The set of outgoing arcs of source states $M_{i} \in$ $M S w h i c h$ do not belong to the set of $\operatorname{arcs} T_{a}\left(M_{i}\right)$ constitute initially the $\mathrm{MC}_{i} \cdot \mathrm{MC}_{1}:\left\{\left(M_{2}\left[t_{2}>M_{4}\right)\right\} ; \quad \mathrm{MC}_{2}\right.$ : $\left\{\left(M_{5}\left[t_{2}>M_{8}\right),\left(M_{5}\left[t_{1}>M_{9}\right)\right\} ; \mathrm{MC}_{3}:\left\{M_{6}\left[t_{2}>M_{9}\right\}\right.\right.\right.$; $\mathrm{MC}_{4}=\left\{M_{10}\left[t_{2}>M_{13}\right\}\right.$.

Note that source states $M_{5}$ and $M_{6}$ share a common forbidden state $M_{9}$ and the fusion of $\mathrm{MC}_{2}$ and $\mathrm{MC}_{3}$ has not happened. Indeed, the transition $t_{1}$ from $M_{5}$ to $M_{9}$ belongs to $T_{a}\left(M_{6}\right)$. Next, Algorithm 4 generates the following PN controllers:

$$
\begin{gathered}
P_{c 1}: M_{01}\left(P_{c 1}\right)=1 ; C\left(P_{c 1}, .\right)=(0,-2,0,0,0,) ; \\
P_{c 2}: M_{02}\left(P_{c 2}\right)=1 ; C\left(P_{c 2}, .\right)=(-2,-2,0,0,0)
\end{gathered}
$$

$P_{c 3}: M_{03}\left(P_{c 3}\right)=1 ; C\left(P_{c 3},.\right)=(0,-2,0,0,0) ; P_{c 4}:$

$M_{04}\left(P_{c 4}\right)=1 ; C\left(P_{c 4},.\right)=(0,-2,0,0,0)$.

Notably, $P_{c 1}, P_{c 3}$ and $P_{c 4}$ are equal. Therefore, the redundant

\begin{tabular}{|c|c|c|c|}
\hline $\begin{array}{l}\mathrm{PN} \\
\text { controller }\end{array}$ & $\begin{array}{c}\text { Marking } \\
\text { no. }\end{array}$ & $\begin{array}{l}\text { Activation } \\
\text { vectors }\end{array}$ & $\begin{array}{l}\text { Deactivation } \\
\text { vectors }\end{array}$ \\
\hline \multirow[t]{2}{*}{$P_{c 1}$} & $M_{2}$ & {$\left[\begin{array}{lllll}0 & 1 & 0 & 0 & 0\end{array}\right]^{T}$} & {$\left[\begin{array}{lllll}0 & 1 & 0 & 0 & 1\end{array}\right]^{T}$} \\
\hline & $M_{3}^{2}$ & {$\left[\begin{array}{lllll}1 & 0 & 0 & 0 & 0\end{array}\right]^{T}$} & {$\left[\begin{array}{llllll}1 & 0 & 0 & 1 & 0\end{array}\right]^{T}$} \\
\hline$P_{c 2}$ & $M_{5}^{3}$ & {$\left[\begin{array}{lllll}1 & 1 & 0 & 0 & 0\end{array}\right]^{T}$} & {$\left[\begin{array}{llll}0 & 0 & 0 & 1\end{array}\right]^{T}$} \\
\hline
\end{tabular}
control places $P_{c 3}$ and $P_{c 4}$ can be removed, and the Boolean control function is $F_{B c}=P_{c 1} P_{c 2}$. The controlled PN model is shown in Figure 10.

\begin{tabular}{lccc} 
Table 1. Information of MC markings. & & \\
\hline $\begin{array}{l}\text { Minimal } \\
\text { cut no. }\end{array}$ & $\begin{array}{c}\text { Marking } \\
\text { no. }\end{array}$ & Classification & $\begin{array}{c}\text { Marking } \\
{\left[p_{1}, p_{2}, p_{3}\right]^{T}}\end{array}$ \\
\hline $\mathrm{MC}_{1}$ & $M_{2}$ & $M S_{1}$ & {$[3,0,1]^{T}$} \\
& $M_{4}$ & $M I_{1}$ & {$[2,0,2]^{T}$} \\
& $M_{5}$ & $M S_{1}$ & {$[2,1,1]^{T}$} \\
& $M_{8}$ & $M I_{1}$ & {$[1,1,2]^{T}$} \\
& $M_{6}$ & $M S_{1}$ & {$[2,2,0]^{T}$} \\
& $M_{9}$ & $M I_{1}$ & {$[1,2,1]^{T}$} \\
& $M_{10}$ & $M S_{1}$ & {$[1,3,0]^{T}$} \\
& $M_{13}$ & $M I_{1}$ & {$[3,3,1]^{T}$} \\
& $M_{3}$ & $M n_{1}$ & {$[2,1,1]^{T}$} \\
$M_{2}$ & $M_{5}$ & $M S_{2}$ & {$[1,2,1]^{T}$} \\
\hline
\end{tabular}

Table 2. Activation and deactivation vectors.

\section{Comparison with previous works}

This section presents three tables with regard to compare our new method with previous works. First, we compare this work with the classical approach of the theory of regions developed by Ghaffari et al. (2002a) in Example 1.

Moreover, this method is confronted in Example 2 with the previous deadlock prevention policies and especially with the recent method of Huang and Pan (2011) that has many advantages and solves problem of forbidden state transition. Indeed, its experimental results with comparisons infer that its policy seems to be the most efficient approach among existing methods such as the research works of Li et al. (2008); Park and Reveliotis (2000); Uzam and Zhou (2007) and Piroddi, Cordone, and Fumagalli (2008). Although, this methods do not consider uncontrollable transitions in $\mathrm{PN}$ models used in experimentation.

Finally, it is shown in Example 3 that the resolution of the problem whenthe PN controller does not exist without resorting to partial PN controllers approach (Ghaffari et al., 2002). For convenience, the two approaches related to the two approaches 
Table 3. Comparison results of Example 1.

\begin{tabular}{lccccc}
\hline Approach & $\begin{array}{c}\text { No. of } \\
\text { monitors }\end{array}$ & $\begin{array}{c}\text { Reachable } \\
\text { markings }\end{array}$ & $\begin{array}{c}\text { No. of event } \\
\text { separation instances } \Omega\end{array}$ & $\begin{array}{c}\text { No. of equations } \\
(\boldsymbol{L}) \text { in all } L \boldsymbol{S}_{\boldsymbol{i}}\end{array}$ & $\begin{array}{c}\text { Computation } \\
\text { Computation time (ms) }\end{array}$ \\
\hline$S_{1}$ & 2 & 6 & 5 & 15 & 219 \\
$S_{2}$ & 2 & 6 & 5 & 13 & 190 \\
$\boldsymbol{G}_{1}$ & 2 & 6 & 5 & 40 & Minimal cuts \\
\hline
\end{tabular}

Table 4. Additional control places.

\begin{tabular}{lccc}
\hline Approach & $\begin{array}{c}\text { Additional } \\
\text { control places }\end{array}$ & $M_{0 i}\left(P_{c i}\right)$ & $C\left(P_{c i},.\right)$ \\
\hline$S_{1}$ & $P_{c 1}$ & 1 & $(-1,-2,0,1,2)$ \\
$S_{2}$ & $P_{c 2}$ & 1 & $(-2,0,0,0,0)$ \\
& $P_{c 1}$ & 1 & $(0,-2,0,0,0)$ \\
$G_{1}$ & $P_{c 2}$ & 1 & $(-2,-2,0,0,0)$ \\
& $P_{c 1}$ & 1 & $(0,-1,0,0,1)$ \\
& $P_{c 2}$ & 3 & $(-1,-2,0,1,2)$ \\
\hline
\end{tabular}

are called $S_{1}$ and $S_{2}$. Moreover, the related research works (Ghaffari, Rezg, \& Xie, 2002a), (Ghaffari et al., 2002), (Huang \& Pan, 2011) are called $G_{1}, G_{2}$ and $H$, respectively.

\subsection{Example 1 (ENIM FMS)}

Table 3 lists the experimental results with the work of $G_{1}$ to synthesise PN controllers for the FMS example. One can observe that for the same number of reachable markings and event separation instances, one can synthesise the same number of maximally permissive supervisors with reduced number of equations $(L)$ reflecting the theory of regions. Indeed, two control places founded with the classical approach of $G_{1}$ are obtained by solving 40 equations with computation time of $593 \mathrm{~ms}$, whereas with MC approaches $L$ is divided by 3 . The reduction technology is involved in $S_{1}$ and $S_{2}$ by reducing systematically the computation time, which decreases from 593 to 219 and $190 \mathrm{~ms}$ for both approaches $S_{1}$ and $S_{2}$. The controlled system net with the three approaches is obtained and shown in Table 4.

As a result, one can infer that our new method translated by $S_{1}$ and $S_{2}$ can drastically reduce the encountered difficulties in solving all equations of the theory of regions.

\subsection{Example $2(\mathrm{H})$}

Example 2 is taken from Huang and Pan (2011), based on Table 5, 4 event separation instances and 46 equations of the theory of regions are necessary to be processed if $H$ is used. On the other side, our new method needs 10 event separations and respectively 22 and 18 equations to solve for approaches $S_{1}$ and $S_{2}$. It is obvious that three control places are used to control this example with the $H$ method against three and
Table 6. Additional control places.

\begin{tabular}{cccc}
\hline A & $\begin{array}{c}\text { Additional } \\
\text { control places }\end{array}$ & $M_{0 i}\left(P_{c i}\right)$ & $C\left(P_{c i},.\right)$ \\
\hline$S_{1}$ & $P_{c 1}$ & 0 & $(-2,0,0,0,0,0,0,0,0,0)$ \\
& $P_{c 2}$ & 0 & $(0,-2,0,0,0,0,0,0,0,0)$ \\
$S_{2}$ & $P_{c 3}$ & 0 & $(0,0,0,0,0,-2,0,0,0,0)$ \\
& $P_{c 1}$ & 1 & $(0,0,0,0,0,0,0,0,-2,0)$ \\
& $P_{c 2}$ & 1 & $(0,0,0,-2,0,0,0,0,0,0)$ \\
& $P_{c 3}$ & 1 & $(0,0,0,0,0,0,0,-2,-2,0)$ \\
$H$ & $P_{c 4}$ & 1 & $(0,0,0,-2,-2,0,0,0,0,0)$ \\
& $P_{c 1}$ & 2 & $(0,0,0,0,1,-1,0,1,-1,0)$ \\
& $P_{c 2}$ & 3 & $(0,0,0,1,-1,0,0,1,-1,0)$ \\
& $P_{c 3}$ & 2 & $(0,0,0,1,-1,0,0,0,1,-1)$ \\
\hline
\end{tabular}

four control places for $S_{1}$ and $S_{2}$. The controlled system net is obtained with the three approaches and shown in Table 6. The main advantage of the algorithms of $S_{1}$ and $S_{2}$ is the gain of computation time which is reduced from 489 to 234 and 191 $\mathrm{ms}$. Indeed, for the same number of reachable markings of 20 states, one can solve only 22 and 18 equations of the theory of regions to synthesise the $\mathrm{PN}$ supervisors comparing to 46 equations for the $H$ method. Another very important factor for the comparison between the three methods is not listed in Table 5. In fact, the $H$ method does not consider uncontrollable transitions in the PN models, which is a weakness in this work. As a result, one can infer that our new method is the most efficient among $H$ methods.

\subsection{Example $3\left(G_{1} G_{2}\right)$}

Example 3 is taken from Ghaffari et al. (2002) which present the forbidden state transitions problem insoluble with pure control places. Indeed, this method solves the problem when a single control place does not exist, and the main idea is to synthesise several control places to prevent a forbidden transition. The set of this control places is called a partial controller. Based on the experiment results in Table 7, 236 equations of the theory of regions to solve for 8 event separation instances are needed if one uses the $G_{2}$ method in this example. Although the reduced technology is involved in $S_{1}$ and $S_{2}$, both the approaches need to handle 16 equations that shows the big reduction of timeconsuming decreasing from 1540 to 110 and 115 ms. Moreover,

Table 5. Comparison results of Example 2.

\begin{tabular}{lcccccc}
\hline Approach & $\begin{array}{c}\text { No. of } \\
\text { monitors }\end{array}$ & $\begin{array}{c}\text { Reachable } \\
\text { markings }\end{array}$ & $\begin{array}{c}\text { No. of event } \\
\text { separation instances } \Omega\end{array}$ & $\begin{array}{c}\text { No. of equations } \\
(\boldsymbol{L}) \text { in all } L S_{\boldsymbol{i}}\end{array}$ & $\begin{array}{c}\text { Computation } \\
\text { time (ms) }\end{array}$ & Method \\
\hline$S_{1}$ & 3 & 20 & 10 & 22 & 234 & Minimal cuts \\
$S_{2}$ & 4 & 20 & 10 & 18 & 191 & Minimal cuts \\
$\mathrm{H}$ & 3 & 20 & 4 & 46 & 489 & Reachability graph based \\
\hline
\end{tabular}


Table 7. Comparison results of Example 3

\begin{tabular}{lccccc}
\hline Approach & $\begin{array}{c}\text { No. of } \\
\text { monitors }\end{array}$ & $\begin{array}{c}\text { Reachable } \\
\text { markings }\end{array}$ & $\begin{array}{c}\text { No. of event } \\
\text { separation instances } \Omega\end{array}$ & $\begin{array}{c}\text { No. of equations } \\
(\boldsymbol{L}) \text { in all } L \boldsymbol{S}_{\boldsymbol{i}}\end{array}$ & $\begin{array}{c}\text { Computation } \\
\text { time (ms) }\end{array}$ \\
\hline$S_{1}$ & 2 & 30 & 8 & 16 & 16 \\
$S_{2}$ & 2 & 30 & 8 & 236 & 110 \\
$\boldsymbol{G}_{2}$ & 5 & 30 & 8 & Minimal cuts & 113 \\
\hline
\end{tabular}

Table 8. Additional control places.

\begin{tabular}{lccc}
\hline Approach $(A)$ & $\begin{array}{c}\text { Additional } \\
\text { control places }\end{array}$ & $M_{0 i}\left(P_{c i}\right)$ & $C\left(P_{c i},.\right)$ \\
\hline$S_{1}$ & $P_{c 1}$ & 0 & $(-2,0,0,0)$ \\
$S_{2}$ & $P_{c 2}$ & 0 & $(0,-2,0,0)$ \\
$G_{2}$ & $P_{c 1}$ & 1 & $(0,-2,0,0)$ \\
& $P_{c 2}$ & 1 & $(-2,0,0,0)$ \\
& $P_{c 1}$ & 4 & $(-1,-1,0,2)$ \\
& $P_{c 2}$ & 7 & $(-2,-1,-2,5)$ \\
& $P_{c 3}$ & 0 & $(1,-3,0,2)$ \\
& $P_{c 4}$ & 9 & $(-3,-2,2,3)$ \\
& $P_{c 5}$ & 0 & $(1,-3,2,0)$ \\
\hline
\end{tabular}

with the $G_{2}$ method, five monitors have been obtained what defines three partial controllers against two PN supervisors with approaches $S_{1}$ and $S_{2}$. The controlled PN model that obtains maximally permissive supervisors with 30 reachable markings is shown in Table 8. Therefore, not only the resolution complexity of the theory of regions is reduced in this method, but the problem of partial controllers defined in Ghaffari et al. (2002) is also solved with a reduced approach. As a result, one can infer that the new approaches $S_{1}$ and $S_{2}$ are the most efficient among the $G_{2}$ method.

\section{Conclusion and future work}

The proposed policy based on MC approaches is implemented in a FMS. The results analysis shows that our control policy is more efficient than the previous work using the theory of regions for control synthesis. Indeed, for the same number of monitors, we can synthesise the PN controllers under fewer equations and computation time. The application of the theory of regions is confined to the $\mathrm{MC}$ and not to all the reachability graph. In fact, it reflects the great reduction of the resolution complexity of this theory. Moreover, our method solves insoluble problems of forbidden state transition problem under the combinatorial explosion of states and the convexity of state equations that may exist in the LS of the theory of regions.

Based on experimental results, it is obvious to notice the considerable reduction of the computational cost of the theory of regions. Our new policy provides an efficient method to obtain the maximally permissive supervisor.

Hence, in the near future, we intend to find other methodologies in order to reduce the resolution complexity of the theory of regions, where it is not necessary to generate the reachability graph to synthesise the PN controller which is an important issue in control synthesis.

\section{Acknowledgements}

The authors would like to thank the International Journal of Systems Science and the reviewers for possible evaluation of our manuscript entitled: Supervisory Control based on Minimal Cuts and Petri Net Sub-Controllers Coordination. The authors would also like to thank everyone who helped complete this work with their continued efforts and support. Sadok Rezig would like to thank Professor Nidhal Rezg for all the valuable recommendations and advices and Associate-Professor Zied Achour for his monitoring and assistance, without whom the author's contributions would not have been brought to a successful completion.

\section{Disclosure statement}

No potential conflict of interest was reported by the authors.

\section{References}

Badouel, E., \& Darondeau, P. (1998). Theory of regions. In W. Reisig \& G. Rozenberg (Eds.), Lectures on Petri Nets I: Basic models (pp. 529-586). Berlin: Springer-Verlag.

Darondeau, Ph., \& Xie, X. (2003). Linear control of live marked graphs. Automatica, 39(3), 429-440.

Ghaffari, A., Njima, F.R., Rezg, N., \& Xie, X. (2002). Design and coordination of partial Petri net controllers for a maximally permissive supervisory control. In IEEE International Conference on Systems Man and Cybernetics-SMC'02. Retrieved from https://hal.archivesouvertes.fr/inria-00100957/

Ghaffari, A., Rezg, N., \& Xie, X. (2002a). Live and maximally permissive controller synthesis using theory of regions. In Synthesis and control of discrete event systems (pp. 155-166). Springer. Retrieved from http://link.springer.com/chapter/10.1007/978-1-4757-6656-1_10

Ghaffari, A., Rezg, N., \& Xie, X. (2002b). Net transformation and theory of regions for optimal control of Petri nets. In IFAC World Congress on Automatic Control. Retrieved from https://hal.archivesouvertes.fr/inria-00100960/

Giua, A., DiCesare, F., \& Silva, M. (1992). Generalized mutual exclusion contraints on nets with uncontrollable transitions. In Systems, Man and Cybernetics, 1992., IEEE International Conference on (pp. 974-979). Chicago, IL: IEEE.

Haoxun, C. (2000). Control synthesis of Petri nets based on S-decreases. Discrete Event Dynamic Systems, 10(3), 233-249.

Huang, Y.-S., \& Pan, Y.-L. (2011). An improved maximally permissive deadlock prevention policy based on the theory of regions and reduction approach. IET Control Theory \& Applications, 5(9), 1069-1078.

Iordache, M.V., \& Antsaklis, P.J. (2007). Petri Net Supervisors for Disjunctive Constraints. In American Control Conference, 2007. ACC '07 (pp. 4951-4956). New York, NY: IEEE.

Iordache, M., Wu, P., Zhu, F., and Antsaklis, P. (2013). Efficient design of Petri-net supervisors with disjunctive specifications. In Proceedings of IEEE International Conference on Automation Science and Engineering. Madison, WI: IEEE.

Li, Z.W., Zhou, M.C., \& Jeng, M.D. (2008). A maximally permissive deadlock prevention policy for FMS based on Petri net siphon control and the theory of regions. IEEE Transactions on Automation Science and Engineering, 5(1), 182-188.

Murata, T. (1989). Petri nets: Properties, analysis and applications. Proceedings of the IEEE, 77(4), 541-580.

Park, J., \& Reveliotis, S.A. (2000). Algebraic synthesis of efficient deadlock avoidance policies for sequential resource allocation systems. IEEE Transactions on Robotics and Automation, 16(2), 190-195. 
Piroddi, L., Cordone, R., \& Fumagalli, I. (2008). Selective siphon control for deadlock prevention in Petri Nets. IEEE Transactions on Systems, Man and Cybernetics, Part A: Systems and Humans, 38(6), 1337-1348.

Qin, M., Li, Z., Zhou, M.C., Khalgui, M., \& Mosbahi, O. (2012). Deadlock prevention for a class of Petri nets with uncontrollable and unobservable transitions. IEEE Transactions on Systems, Man and Cybernetics, Part A: Systems and Humans, 42(3), 727-738.

Ramadge, P.J., \& Wonham, W.M. (1987). Supervisory control of a class of discrete event processes. SIAM Journal on Control and Optimization, 25(1), 206-230.

Ramadge, P.J.G., \& Wonham, W.M. (1989). The control of discrete event systems. Proceedings of the IEEE, 77(1), 81-98.

Rezg, N., Rezig, S., Kammoun, M.-A., \& Achour, Z. (2014). Control synthesis for admissible paths based on theory of regions: Application to a flexible manufacturing system. In 2014 IEEE Conference on Control Applications (CCA) (pp. 1807-1811). Juan Les Antibes: IEEE.

Rezig, S., Achour, Z., \& Rezg, N. (2014). Control synthesis based on reachability graph with minimal cuts: Application to a flexible manufacturing system. In 2014 IEEE Emerging Technology and Factory Automation (ETFA). Barcelona: IEEE.

Rezig, S., Achour, Z., Rezg, N., \& Kammoun, M.-A. (2014). Optimal control synthesis for a flexible manufacturing system based on minimal cuts. In 2014 IEEE International Conference on Industrial Engineering and Engineering Management (IEEM) (254-258). Bandar Sunway: IEEE.

Ru, Y., Cabasino, M. P., Giua, A., \& Hadjicostis, C.N. (2012). Supervisor synthesis for discrete event systems under partial observation and arbitrary forbidden state specifications. Discrete Event Dynamic Systems, 24(3), 275-307.

Uzam, M. (2009). On suboptimal supervisory control of Petri nets in the presence of uncontrollable transitions via monitor places. The International Journal of Advanced Manufacturing Technology, 47(5-8), 567579 .

Uzam, M., \& Zhou, M. (2007). An iterative synthesis approach to Petri netbased deadlock prevention policy for flexible manufacturing systems. IEEE Transactions on Systems, Man and Cybernetics, Part A: Systems and Humans, 37(3), 362-371. 